Multi-machine analysis of termination scenarios with comparison to simulations of controlled shutdown of ITER discharges

de Vries, P.C.

2018

de Vries , P C , JET Contributors \& Ahlgren , T 2018, ' Multi-machine analysis of termination scenarios with comparison to simulations of controlled shutdown of ITER discharges ' , Nuclear Fusion , vol. 58 , no. 2 , 026019 . https://doi.org/10.1088/1741-4326/aa9c4c

http://hdl.handle.net/10138/306774

https://doi.org/10.1088/1741-4326/aa9c4c

publishedVersion

Downloaded from Helda, University of Helsinki institutional repository.

This is an electronic reprint of the original article.

This reprint may differ from the original in pagination and typographic detail.

Please cite the original version. 
PAPER

\section{Multi-machine analysis of termination scenarios with comparison to simulations of controlled shutdown of ITER discharges}

To cite this article: P.C. de Vries et al 2018 Nucl. Fusion 58026019

View the article online for updates and enhancements.
Related content

- Simulation of profile evolution from ramp-
$\frac{\text { up to ramp-down and optimization of }}{\text { tokamak plasma termination with the }}$
$\frac{\text { RAPTOR code }}{\text { A A Teplukhina, O Sauter, F Felici et al. }}$
- The effect of the isotope on the H-mode
density limit
A. Huber, S. Wiesen, M. Bernert et al.
- Plasma vertical stabilisation in ITER
Y. Gribov, A. Kavin, V. Lukash et al.

Recent citations

- Overview of the JET preparation for
$\frac{\text { deuterium-tritium operation with the ITER }}{\text { like-wall }}$
E. Joffrin et al
- Progress in disruption prevention for ITER
E.J. Strait et al
- lon cyclotron resonance heating scenarios
$\frac{\text { for DEMO }}{\text { D. Van Eester et al }}$

\section{IOP ebooks}

Bringing you innovative digital publishing with leading voices to create your essential collection of books in STEM research. Start exploring the collection - download the first chapter of every title for free. 


\title{
Multi-machine analysis of termination scenarios with comparison to simulations of controlled shutdown of ITER discharges
}

\author{
P.C. de Vries ${ }^{1}$, T.C. Luce ${ }^{2}$,Y.S. Bae ${ }^{3}$, S. Gerhardt ${ }^{4}$, X. Gong ${ }^{5}$,Y. Gribov ${ }^{1}$, \\ D. Humphreys ${ }^{2}$, A. Kavin ${ }^{6}$, R.R. Khayrutdinov ${ }^{7}$, C. Kessel ${ }^{4}$, S.H. Kim ${ }^{1}$, \\ A. Loarte ${ }^{1}$, V.E. Lukash 7 , E. de la Luna ${ }^{8,9}$, I. Nunes ${ }^{8,10}$, F. Poli ${ }^{4}$, J. Qian ${ }^{5}$, \\ M. Reinke ${ }^{11}$, O. Sauter ${ }^{12}$, A.C.C. Sips ${ }^{8,13}$, J.A. Snipes ${ }^{1}$, J. Stober ${ }^{14}$, \\ W. Treutterer ${ }^{14}$, A.A. Teplukhina ${ }^{12}$, I. Voitsekhovitch ${ }^{15}$, M.H. Woo ${ }^{3}$, S. Wolfe ${ }^{16}$, \\ L. Zabeo ${ }^{1}$, the Alcator C-MOD team ${ }^{16}$, the ASDEX Upgrade team ${ }^{14}$, the \\ DIII-D team ${ }^{2}$, the EAST team ${ }^{5}$, JET contributors ${ }^{\text {a }}$, the KSTAR team ${ }^{3}$, the \\ NSTX-U team ${ }^{4}$ and the TCV team ${ }^{12}$ and ITPA IOS members and experts \\ ${ }^{1}$ ITER Organization, Route de Vinon sur Verdon, 13067 St Paul Lez Durance, France \\ 2 General Atomics, PO Box 85608, San Diego, CA 92186-5608, United States of America \\ 3 National Fusion Research Institute, Daejeon, Republic of Korea \\ ${ }^{4}$ Princeton Plasma Physics Laboratory, Princeton University, Princeton, NJ 08543, \\ United States of America \\ 5 Institute of Plasma Physics, Chinese Academy of Sciences, Hefei 230031, People's Republic of China \\ 6 D.V. Efremov Institute of Electrophysical Apparatus, Saint Petersburg, Russian Federation \\ 7 National Research Center Kurchatov Institute, Moscow, Russian Federation \\ 8 EUROfusion Consortium, JET, Culham Science Centre, Abingdon, OX14 3DB, United Kingdom \\ 9 Laboratorio Nacional de Fusion, CIEMAT, 28040 Madrid, Spain \\ ${ }^{10}$ Associação EURATOM-IST, Instituto de Plasmas e Fusão Nuclear, Lisboa, Portugal \\ 11 Oak Ridge National Laboratory, 1 Bethel Valley Rd., Oak Ridge, TN 37830, United States of America \\ 12 École Polytechnique Fédérale de Lausanne, Swiss Plasma Center (SPC), CH-1015, Switzerland \\ 13 European Commission, Brussels, Belgium \\ 14 Max-Planck-Institut für Plasmaphysik, 85748 Garching, Germany \\ 15 CCFE, Culham Science Centre, OX14 3DB Abingdon, United Kingdom \\ 16 Plasma Science and Fusion Center, MIT, Cambridge, MA, United States of America \\ E-mail: Peter.Devries@iter.org
}

Received 9 July 2017, revised 31 October 2017

Accepted for publication 22 November 2017

Published 22 December 2017

\begin{abstract}
To improve our understanding of the dynamics and control of ITER terminations, a study has been carried out on data from existing tokamaks. The aim of this joint analysis is to compare the assumptions for ITER terminations with the present experience basis. The study examined the parameter ranges in which present day devices operated during their terminations, as well as the dynamics of these parameters. The analysis of a database, built using a selected set of experimental termination cases, showed that, the H-mode density decays slower than the plasma current ramp-down. The consequential increase in $f_{\mathrm{GW}}$ limits the duration of the $\mathrm{H}$-mode phase or result in disruptions. The lower temperatures after the drop out of $\mathrm{H}$-mode will allow the plasma internal inductance to increase. But vertical stability control remains manageable in ITER at high internal inductance when accompanied by a strong elongation reduction. This will result in ITER terminations remaining longer at low $q\left(q_{95} \sim 3\right)$ than most present-day devices during the current ramp-down. A fast power ramp-down leads to a larger change in $\beta_{\mathrm{p}}$ at the $\mathrm{H}-\mathrm{L}$ transition, but the experimental data showed that these
\end{abstract}

${ }^{\text {a }}$ See Litaudon et al [28]. 
are manageable for the ITER radial position control. The analysis of JET data shows that radiation and impurity levels significantly alter the $\mathrm{H}-\mathrm{L}$ transition dynamics. Self-consistent calculations of the impurity content and resulting radiation should be taken into account when modelling ITER termination scenarios. The results from this analysis can be used to better prescribe the inputs for the detailed modelling and preparation of ITER termination scenarios.

Keywords: plasma control, termination scenario, tokamak operation, ITER

(Some figures may appear in colour only in the online journal)

\section{Introduction}

The controlled shutdown is an often overlooked, though important, phase of the tokamak discharge. The dynamics during this phase complicate control, making it difficult to avoid operational limits, which in the worst case, may lead to a disruption. This is exacerbated by the fact that at the end of the discharge, the device is usually operating close to its technical limits. For unplanned terminations, triggered by offnormal events, the situation complicates further. The ability to carry out a well-controlled termination contributes significantly to the avoidance of disruptions.

To improve our understanding of the dynamics and control of ITER terminations, a study has been carried out on data from existing tokamaks. The aim of this joint analysis is to compare the assumptions for ITER terminations with the present experience basis. The study examined the parameter ranges in which present day devices operated during their terminations, as well as the dynamics of these parameters. The dynamics may vary considerably over the duration of the termination; hence, simply comparing average values may not always be sufficient. Moreover, the dynamics of different parameters are sometimes coupled. The analysis addresses changes in plasma shape and internal inductance, $\ell_{\mathrm{i}}$, during the plasma current ramp-down, relevant to vertical stability (VS) control, the energy (or, the poloidal $\beta$ : $\beta_{\mathrm{p}}$ ) decay, which relates to the radial position control, and the controllability of both the density decay and the $\mathrm{H}$ to L-mode back transition. Typical time scales, such as the energy confinement time and the $L / R$ time (where $L$ is the plasma column inductance and $R$ its average resistance) do not have a fixed ratio from device to device, complicating the extrapolation of the termination scenario results.

This paper will first describe, in section 2, the specifics of discharge terminations in ITER, giving the main restrictions and control aspects. In section 3, the database of selected terminations from Alcator C-Mod, ASDEX Upgrade, DIII-D, EAST, JET, KSTAR, NSTX/NSTX-U and TCV is presented. The database also contains simulated ITER terminations. This is followed by a comparison of the stability aspects, in section 4, and dynamics of a number of key parameters, in section 5. Section 6 discusses the power balance during the termination, the general behavior of the radiative power and its influence on the LH transition. In the final section, section 7 , the conclusions are summarized and discussed. The results from the joint analysis can be used to better prescribe the inputs for the modelling and to aid the further preparation of ITER termination scenarios.

\section{ITER termination scenarios: restrictions and example}

The termination phase should achieve a simultaneous rampdown of the plasma current, kinetic energy and particle density while maintaining control over the radiation levels, plasma position and shape (i.e. avoid overheating the first wall) and VS, staying within the capabilities of the poloidal field coils and power supplies and heating systems. Stability boundaries and general operational limits must also be avoided. ITER will operate at high densities and a controlled density decay is important to avoid the Greenwald density limit and uncontrolled detachment towards the end of the current ramp-down, while also managing the $\mathrm{H}-\mathrm{L}$ transition timing and exit from fusion burn. Avoiding the detachment limit might be a more relevant limit, than the Greenwald density limit, in L-mode at lower currents. ITER power supply limitations and the thick vessel slow the control response for VS and the radial position. Previously, experiments on discharge terminations focused mainly on the electromagnetic changes and on the controllability of VS [1-3]. It was found that VS control could be maintained in ITER by restricting the increase in internal inductance $\ell_{\mathrm{i}}$ (e.g. by staying in H-mode and keeping the temperature high) and reducing the elongation, $\kappa$. Changes to the shape are obviously restricted by the PF coil limits but for large elongation changes the power flow to the upper part of the blanket and the position of upper strike points need to be controlled. In ITER, plasmas heated by auxiliary power should remain diverted because, at currents of $I_{\mathrm{p}} \sim 7.5 \mathrm{MA}$ or above, the blanket can only sustain Ohmic power for a short time ( $\sim$ a few secs). A fast drop in $\beta_{\mathrm{p}}$ during the H-L transition, may result in an uncontrolled inward radial motion. This means the plasma could touch the inner wall or become less vertically stable as it loses its proximity to the vessel.

There is no single solution to overcome these issues for ITER terminations. The design of a termination scenario can place different weights on each constraint, e.g. reducing the plasma volume allowing a larger radial excursion, hence a larger drop in $\beta_{\mathrm{p}}$. These weights also depend on the goal of the termination. A normal ITER termination should aim to be in full control until the current is below $I_{\mathrm{p}}=3 \mathrm{MA}$, when the direct disruption impact is expected to be benign [4], and even lower when runaways are considered. The goal for an 


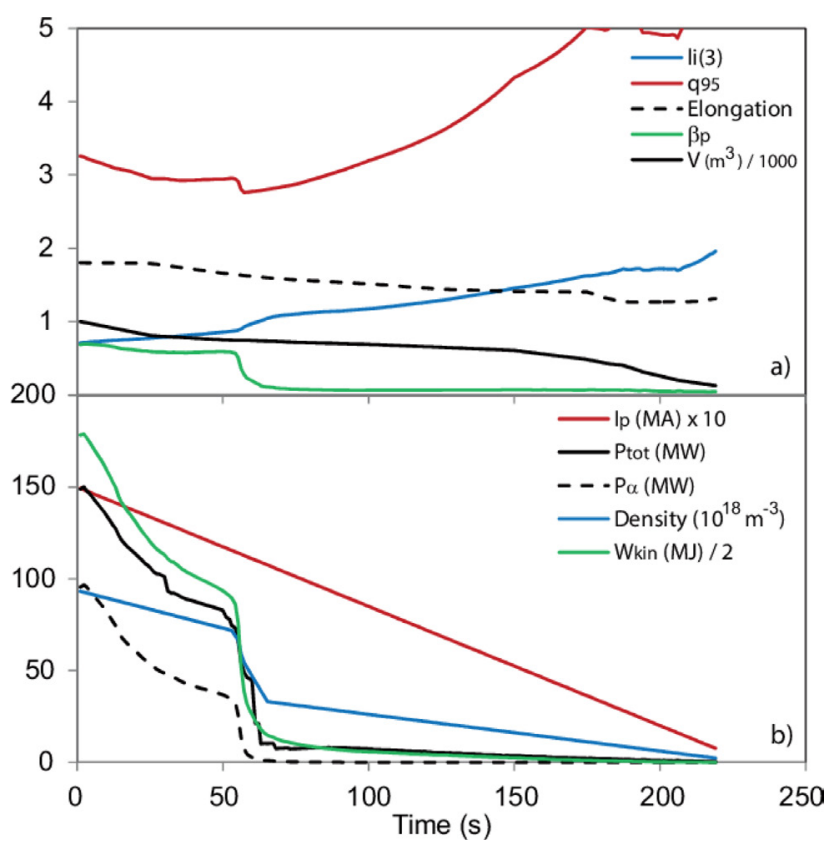

Figure 1. A modelled ITER termination (Corsica Hmode_15MA_13) from $I_{\mathrm{p}}=15 \mathrm{MA}$ at full performance $\left(W_{\text {kin }}=350 \mathrm{MJ}, P_{\alpha}=100 \mathrm{MW}\right)$ taking approximately $220 \mathrm{~s}$. (a) Time traces of the internal inductance, $\ell_{\mathrm{i}}$, the $q_{95}$, the elongation, $\kappa$, the poloidal $\beta$, and volume (in units of $1000 \mathrm{~m}^{3}$ ). (b) The plasma current, $I_{\mathrm{p}}$, (in units of $100 \mathrm{kA}$ ), the total input power, $P_{\text {tot }}$, the $\alpha$-heating power, $P_{\alpha}$, the density (in $10^{18} \mathrm{~m}^{-3}$ ) and the kinetic energy, $W_{\text {kin. }}$. The H-L back transition is visible as fast drop in $\beta_{\mathrm{p}}$ and $W_{\mathrm{kin}}$, around $t=60 \mathrm{~s}$.

emergency termination is different, aiming for a fast reduction of current and energy, with the knowledge that control will be lost and the plasma may disrupt, although with a smaller impact [5]. Studies have also been carried out on the fastest ITER exits from full performance, i.e. by direct switch off of auxiliary heating [6]. The fastest ITER current ramp-down is limited by the PF coil voltages and the requirements to control shape, position and VS stability with a certain precision. In ITER, a fully controlled current ramp-down from $I_{\mathrm{p}}=15 \mathrm{MA}$ to below $I_{\mathrm{p}}=1 \mathrm{MA}$ can be achieved in $\sim 60 \mathrm{~s}$. An example of a typical ITER termination, modelled with the Corsica code $[7,8]$, is shown in figure 1 . The current ramp-down has a duration of $210 \mathrm{~s}$, with a moderate current ramp-down rate of $0.07 \mathrm{MA} \mathrm{s}^{-1}$. At the start of the termination the plasma is still in H-mode and at full performance, with an $\alpha$-power of $P_{\alpha}=100 \mathrm{MW}(Q=10)$ and a kinetic energy of $W_{\text {kin }}=350$ MJ. The heating power decays relatively quickly, mainly due to the fast decrease in $\alpha$-power as the current is reduced.

The H-L transition already takes place $60 \mathrm{~s}$ after the start of the termination. The model has to make an assumption for the duration of this transition, here less than 10 s. Similarly the decay of the density is assumed such that the Greenwaldfraction remains constant, with a jump down at the time of the $\mathrm{H}-\mathrm{L}$ transition. Figure 1(a), shows the rather large drop in $\beta_{\mathrm{p}}$ at the time of this transition which in this case avoids a large radial movement that would cause the plasma to contact the wall. Especially during the L-mode phase, $\ell_{\mathrm{i}}$ increases considerably, but VS is ensured by a reduction in plasma volume in the first $20 \mathrm{~s}$ of the termination followed by a steady reduction of $\kappa$ and volume. In this example, the volume is reduced by $25 \%$ and elongation reduced to $\kappa=1.68$ in the first $25 \%$ of the current ramp-down. The elongation is further reduced to, $\kappa=1.5$ at $44 \%$ of the current ramp-down. The volume reduction also allows for a larger radial movement at the time of the $\mathrm{H}-\mathrm{L}$ transition. As a consequence of this volume change, $q_{95}$ remains around 3 for almost half of the current ramp-down (i.e. up to $t \sim 100 \mathrm{~s}$ ), only to increase afterwards.

\section{Database of tokamak terminations}

A database has been created consisting of typical, special and ITER-like terminations from Alcator C-Mod, ASDEX Upgrade, DIII-D, EAST, JET, KSTAR, NSTX/NSTX-U and TCV. Hence, there are examples from devices with full metal walls that can be compared with those with carbon walls, and two devices that, like ITER, have super-conducting coils. For JET both carbon wall and metal wall cases have been included. Wide ranges of heating schemes were used in the database terminations. DIII-D, JET and TCV provided also ohmic terminations, although the emphasis of the analysis presented in this paper is on the termination from H-mode. It should be noted that the database only comprises a small selection discharges that do not necessarily span the full capabilities and parameter ranges at each device. The details of the different discharges and modelled terminations in the database are summarized in table 1.

These can be directly compared with four modelled ITER terminations; one termination with a moderate duration (shown in figure 1), modelled with Corsica [7, 8] two fast terminations modelled by the DINA code [9] with a duration of 60 and $68 \mathrm{~s}$, and finally, a DINA simulation of a hypothetical slowest possible termination, in which the plasma current decays naturally while being kept in H-mode, lasting $1100 \mathrm{~s}$. In the paper, this entry is identified, separately from the other ITER cases, by a black border around its orange diamond. For some database entries, certain parameters were not provided, therefore, these are missing from some figures in the paper.

In figure 2, a comparison of a number of characteristic time-scales is presented. The average current ramp-down for each database entry is shown, in figure $2(a)$, as a function of the inductance, $L$, for each device, here calculated as: $\mu_{o} r_{o}\left(\ln \left(8 r_{o} / a\right)-2-\ell_{\mathrm{i}} / 2\right)$, where $r_{o}$ and a are the major and minor radius, respectively, and assuming $\ell_{\mathrm{i}}=1$. The average ramp-down time, $\Delta \tau_{X}$ of a parameter $X$, is here defined as:

$$
\Delta \tau_{X} \equiv \frac{2}{X_{o}} \int X(t) \mathrm{d} t
$$

i.e. the time integrated value of the parameter divided by half the value at the start of the termination.

Figure 2(b) shows that current ramp-down times in the database for larger devices are generally a smaller fraction of the $L / R$ time (with $R$ the plasma resistance), $\tau_{L / R}$, here averaged over the first half of the current decay. This means that standard ramp-downs for the devices in the database follow this trend but does not imply that smaller devices cannot rampdown the current faster or ITER can ramp-down slower than 
Table 1. Summary of the ITPA IOS termination database. In column 1: the device name; in column 2: the number of entries with L-mode and Ohmic terminations in brackets (note that distinction between H-mode, L-mode and Ohmic is here made depending on the state of the discharge at the start of the current-ramp-down, not if the ramp-down is predominantly in done in this state), column 3: major radius, $\mathrm{r}_{\mathrm{o}}$; in column 4: the label for this device used through-out the paper; in column 5: the list of pulse or model identification numbers, with the Ohmic terminations in brackets and the NSTX upgrade entry in italic, to differentiate from the NSTX entries. For JET both carbon wall (CW) and full metal, ITER-like, wall (ILW) cases are included, as indicated in column 5.

\begin{tabular}{|c|c|c|c|c|}
\hline Device & No. Pulses & $r_{o}(\mathrm{~m})$ & Symbol & Pulse or model identifier \\
\hline Alcator C-Mod & 9 & 0.68 & $\mathbf{\square}$ & $\begin{array}{l}\text { 1101210008, 1101210011, 1101210022, } 1101215026, \\
1110104035,1120717021,1120917033,1140408025, \\
1140515012 .\end{array}$ \\
\hline ASDEX upgrade & 4 & 1.65 & $\mathrm{O}$ & $22080,25721,31146,31673$ \\
\hline DIII-D & $2(+1)$ & 1.67 & $\Delta$ & (140408), 140406, 157458 \\
\hline EAST & 2 & 1.85 & $\diamond$ & 54497,54501 \\
\hline ITER & 4 & 6.2 & $>$ & $\begin{array}{l}\text { Corsica: Hmode_15MA_13, } \\
\text { DINA: 15MA-DT-DINA2013-02, } \\
\text { DINA: 15MA-DT-DINA2013-03, } \\
\text { DINA: 15MA-DT-DINA2012-05 }\end{array}$ \\
\hline JET & $10(+4)$ & 2.95 & $\Delta$ & $\begin{array}{l}\text { ILW: 87384, 90651, 90652, 90653, } \\
\text { CW: 74406, 72251, 72249, 74405, 74404, 72209, (72462, } \\
\text { 72459, 72458, 72204) }\end{array}$ \\
\hline KSTAR & 1 & 1.8 & $\square$ & 13466 \\
\hline NSTX upgrade & $2(+2)$ & $0.70(0.64)$ & $\Delta$ & $204179,133110,(132474,133014)$ \\
\hline TCV & $1(+2)$ & 0.22 & O & $51213,(53896,53897)$ \\
\hline
\end{tabular}

the trend. The current ramp-down duration is nearly the $L / R$ time for the modelled ITER case with a natural current decay. The resistance for each entry was determined as $P_{\mathrm{ohmic}} / I_{\mathrm{p}}^{2}$, except for TCV for which Spitzer resistivity is assumed, using temperature and $Z_{\text {eff }}$ data. The $L / R$ time, $\tau_{L / R}$, is the characteristic time of a natural plasma current decay. This differs from the resistive time, $\tau_{R}$, being the typical time scale to achieve an equilibrium internal current density, thus related to changes in $\ell_{\mathrm{i}}[10,11]$, as will be discussed in section 5 .

Figure 2(c) shows the average input power ramp-down time, normalized to that of the current. Those terminations in present day devices, carried out to show how to limit the increase in $\ell_{i}$, have relatively long power ramp-downs, i.e. the ratio with the current ramp-down is $>0.8$. But, for typical ITER terminations, the power ramp-down, and consequently the decay in thermal energy, is relatively fast. The reason is that a large fraction of the heating is due to $\alpha$-power. It is therefore not easy to maintain H-mode over a large part of the current decay in ITER. Of course this differs for ITER terminations with a smaller fraction of $\alpha$-power, for example with lower current scenarios or in the non-activation phase of ITER operations. The average ramp-down of the density shows (figure $2(d)$ ) that this is relatively slow compared to the current ramp-down, for most entries. The reasons and implications of this are discussed in more detail in section 5 .

The average ramp-down times allow only a rough comparison but do not capture the variation in dynamics that may take place during a termination, such as a fast $\mathrm{H}-\mathrm{L}$ back transition. For this purpose one can simply plot values of interest at each time step. Each termination in the database contains data with approximately 150-200 time steps. This has been used to create figure 3 , comparing the energy confinement time, $\tau_{\mathrm{E}}$, and $\tau_{L / R}$. The variation in $\tau_{\mathrm{E}}$, is limited, per device, but also over the duration of the termination, while, $\tau_{L / R}$, often varies over several orders of magnitude. Therefore, it is difficult to match a single value of $\tau_{L / R}$ to each termination case. Figure 3 also shows that ratio of $\tau_{\mathrm{E}}$ to $\tau_{L / R}$ varies from device to devices, leading to a different scaling of kinetic and electromagnetic dynamics.

\section{Comparison of stability aspects}

Maintaining VS is an important aspect for a termination. The VS of the plasma depends on a complex function of $\ell_{\mathrm{i}}, \beta_{\mathrm{p}}$ and elongation $\kappa$, and, furthermore, on the proximity of the plasma to stabilizing passive components, such as the vacuum vessel in ITER, and on the capability of the VS control circuit. The latter factors differ from device to device and this does not make a comparison straightforward. Figure 4(a) show the typical traces in $\kappa$, and $\ell_{\mathrm{i}}$ space that each database entry follows during a termination. The reduction in $\kappa$ that is applied by the ITER cases is relatively large and its data points lie on the edge of the space spanned by the other devices. The complex relationship between vertical instability $\ell_{\mathrm{i}}, \beta_{\mathrm{p}}, \kappa$, can be expressed by the so-called marginal stability parameter, defined as $[12,13]$ :

$$
m_{s}=\left[\frac{1.47\left(1+\mathrm{e}^{-2 l_{\mathrm{i}}+1}\right)}{2(\kappa-1.13)}-1\right]\left(1+0.6\left(\beta_{\mathrm{p}}-0.1\right)\right)
$$

The lower $m_{s}$ is, the more unstable the plasma, although the critical point for VS is device specific. Figure $4(b)$, shows the values of $m_{s}$ during the terminations in the database. In most cases, but not all, the value increases with time (i.e. becomes more stable). The minimum value, at which VS is lost varies from device to device. In ITER it depends on the VS control circuits that are used, ranging from roughly $m_{s}$ $\sim 0.15$ to $\sim 0.25$ when, respectively, VS3 (also using in-vessel 

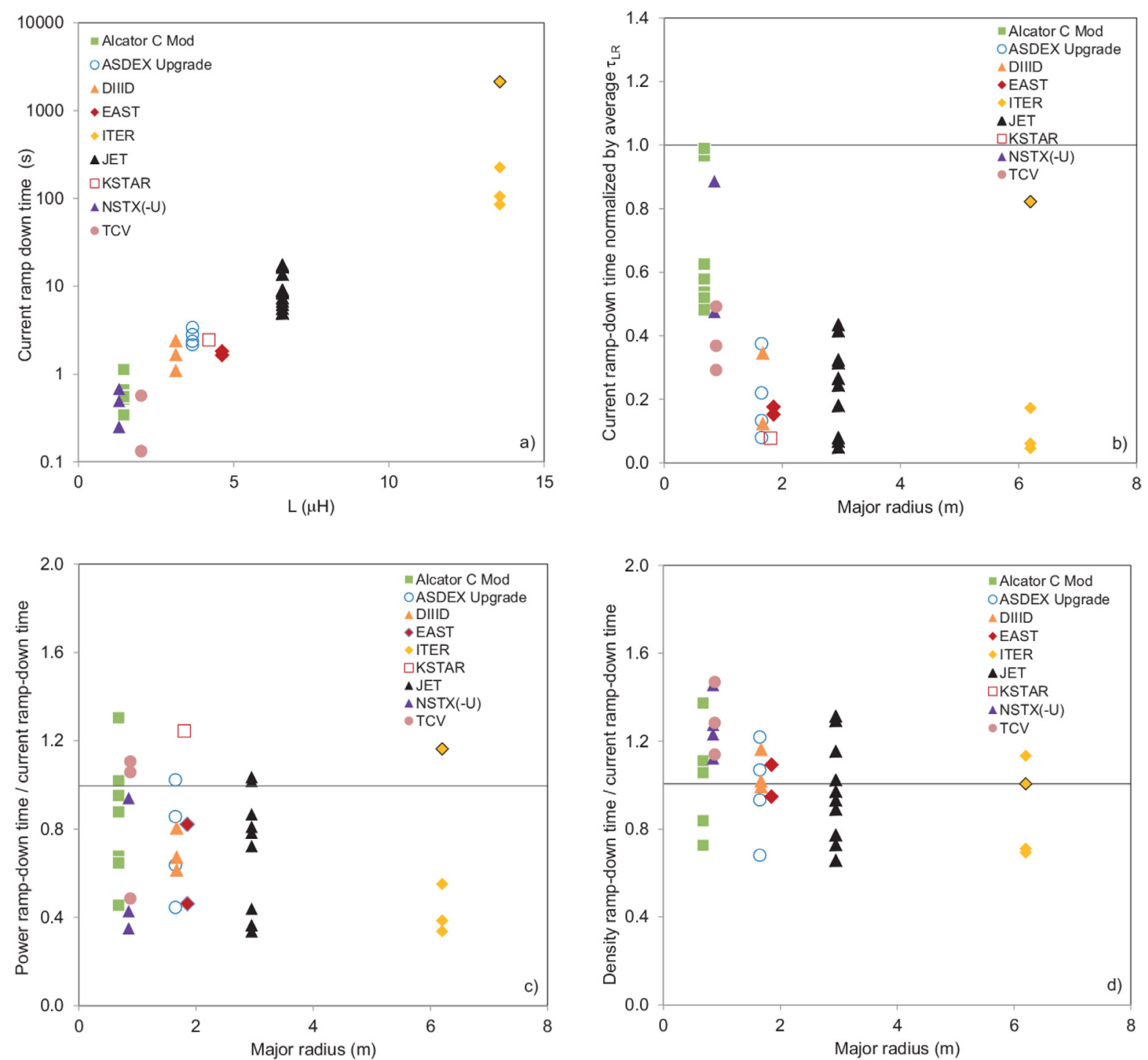

Figure 2. Existence diagrams of database entries showing $(a)$ the current ramp-down time versus the device inductance, $L,(b)$ the current ramp-down time normalized to $\tau_{L / R}$, versus major radius $(c)$ the relative power ramp-down time versus major radius. $(d)$ The relative density ramp-down versus major radius.

coils) or only VS1 (only the ex-vessel coils) are used. The modelled ITER cases often fall near the edge of the experimental cases, because these assume a faster and larger reduction in $\kappa$ than used by most devices. For the more standard ITER terminations, $m_{s}>0.8$, although the slow natural current decay in H-mode achieves a minimum value $m_{s} \sim 0.6$, because the plasma shape is not modified in this case. Except for the very low values found for highly elongated NSTX terminations, lowest values in standard aspect ratio devices are always found above $m_{s} \sim 0.4$. The marginal stability parameter (equation (2)) can be used as an indication if VS can be achieved for ITER modelled terminations, although only selfconsistent modelling of VS control for those scenarios will provide a definite answer.

The well-known $\ell_{\mathrm{i}}-q$ stability diagram, shown in figure 5, provides another view into the stability of the termination. The result of the fast reduction in $\kappa$ and volume is that the modelled ITER terminations remain much longer at $q_{95} \sim 3$, as shown earlier in figure 1, and thereafter trace the upper boundary spanned by the experimental data. This does not necessarily mean that this track is more unstable. However, other devices often show an earlier increase to higher $q_{95}$, which is especially true for those that keep the magnetic field constant during the current ramp-down, such as ASDEX Upgrade, TCV and super-conducting devices, EAST and K-STAR. The faster route to a higher $q_{95}$ might be better with respect to MHD stability, but requires additional heating to avoid excessive $\ell_{\mathrm{i}}$.

\section{Comparison of dynamics}

While in most cases the current is ramped down at a constant rate, the decay rates of thermal energy, or $\beta_{\mathrm{p}}$, density or Greenwald fraction $f_{\mathrm{GW}}$ will vary. Here $f_{\mathrm{GW}}$ is the average density (in $10^{20} \mathrm{~m}^{3}$ ) normalized by the Greenwald density $n_{\mathrm{GW}}=I_{\mathrm{p}} / \pi a^{2}$ with $I_{\mathrm{p}}$ in MA and $a$ in m) [14]. The decay of these parameters will differ between the $\mathrm{H}$ and L-mode phase, and fast changes are expected during the $\mathrm{H}-\mathrm{L}$ transition itself. 


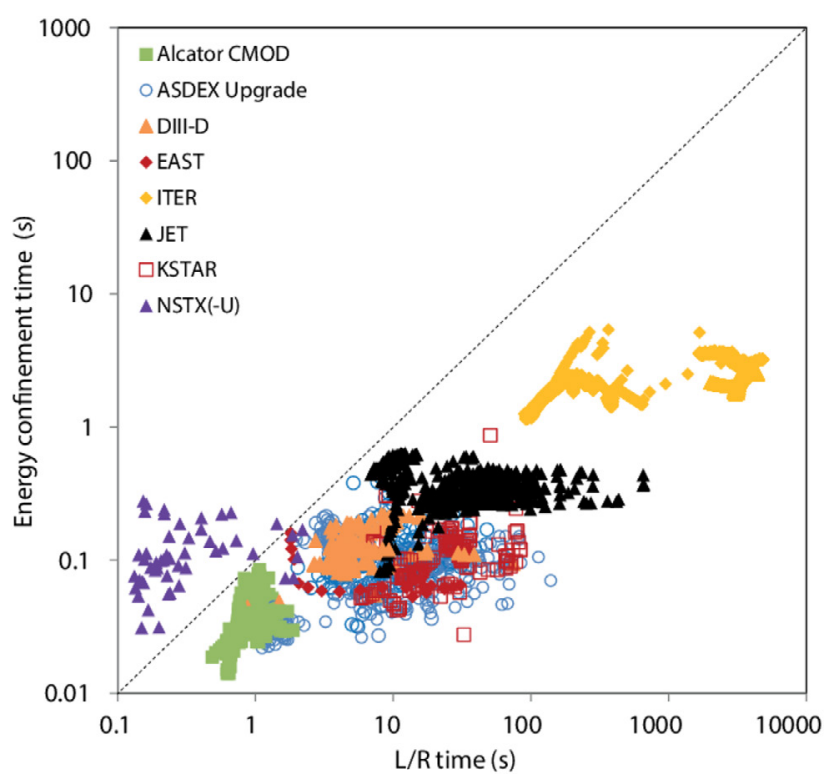

Figure 3. The energy confinement time, $\tau_{\mathrm{E}}$, versus the $L / R$ time, $\tau_{L / R}$, for each time step during a number of database terminations in devices of different size. The trend deviates from a linear scaling, indicated by the dashed line.

The dynamics of a parameter $X$, at a certain time, $t$, during the termination, can be expressed by the decay time, defined as:

$$
\tau_{X} \equiv-X(t) /(\mathrm{d} X(t) / \mathrm{d} t) .
$$

This decay time needs to be determined over a time window, here always chosen to be of the order of the energy confinement time. The decay time differs from the ramp-down time, defined by equation (1). The ramp-down time provides an average value for the parameter decay for the entire termination, while the decay time, allows us to study fast changes in the dynamics, although, with as a consequence a larger error. Figure 6 shows one of the JET terminations from the database. The plasma current is ramp-down from $I_{\mathrm{p}}=2.7 \mathrm{MA}$ at a rate of $0.3 \mathrm{MA} \mathrm{s}^{-1}$. It applies auxiliary power (neutral beam heating) up to $40 \%$ of the current ramp-down and maintains the plasma in $\mathrm{H}$-mode during that time. It shows $\tau_{L / R}$ being several orders of magnitudes longer than the energy confinement time, $\tau_{\mathrm{E}}$. The current, density and energy decay time (i.e. $\tau_{\text {Ip }}, \tau_{n}$ and $\tau_{W}$, respectively) are all roughly comparable but not identical. Variations are visible, especially during the $\mathrm{H}-\mathrm{L}$ transition, when $\tau_{W}$ decreases and briefly equals $\tau_{\mathrm{E}}$.

The analysis shown in figure 6 can be done for all terminations in the database. In figure 7 , the decay time of the energy and density is compared with that of the current for all devices. It shows that at times, the energy and especially density decay slower than the current, such that one obtains an increasing $\beta_{\mathrm{p}}\left(\propto W / \mathrm{I}_{\mathrm{p}}^{2}\right)$ and $f_{\mathrm{GW}},\left(\propto n / I_{\mathrm{p}}\right)$. The fastest energy and density decay, usually of the order of the energy confinement time, are found at the time of the $\mathrm{H}-\mathrm{L}$ transition. Note that negative decay times (i.e. increases) are also possible but not visible in figure 7 .

\subsection{The energy and density decay in $\mathrm{H}$-mode}

The energy and density decay prior to the $\mathrm{H}-\mathrm{L}$ transition are usually slower than the current decay during this stage of the termination. This leads, as for example shown in figure $6(b)$, to an increase in $\beta_{\mathrm{p}}$ and $f_{\mathrm{GW}}$ up to the time of the $\mathrm{H}-\mathrm{L}$ transition. This feature is found in most terminations as can be seen in figure 8. Both parameters tend to increase up to the $\mathrm{H}-\mathrm{L}$ transition. The longer the power is maintained, and the plasma is kept in H-mode, the larger the increase in $\beta_{\mathrm{p}}$ and $f_{\mathrm{GW}}$. The energy decay (i.e. $\beta_{\mathrm{p}}$ ) could be controlled better by feedback control of the auxiliary heating system. But the control of the density decay is more difficult and too high values of $f_{\mathrm{GW}}$ could lead to a disruption. No indication was found that the heating mixture matters with respect to the density decay. The auxiliary power composition varied for the database entries with some being purely neutral beam (NB) heated, others solely by radiofrequency (RF) heating, and some by a mixture of the two. Obviously, when the termination is started at an already high $f_{\mathrm{GW}}$, one cannot keep the plasma in $\mathrm{H}$-mode and simultaneously ramp-down the current for too long, before reaching the Greenwald density limit [14]. In the example shown in figure 6, after the $\mathrm{H}-\mathrm{L}$ transition, in the L-mode phase of the termination, the energy and density decay times are generally shorter than the current decay and $f_{\mathrm{GW}}$ and $\beta_{\mathrm{p}}$ decrease in time (as seen in figure $6(b)$ ), although the opposite is seen in other database entries.

\subsection{The $H-L$ transition}

The magnitude and duration of the change in $\beta_{\mathrm{p}}$ and $f_{\mathrm{GW}}$ at the $\mathrm{H}-\mathrm{L}$ transition will affect the ability to control the radial position of the plasma in ITER. The drop in energy, $\beta_{\mathrm{p}}$, and $f_{\mathrm{GW}}$ over the $\mathrm{H}-\mathrm{L}$ transition were determined by calculating their peak derivatives normalized to the average energy confinement time. Figure $6(d)$ shows these normalized derivatives for the JET example which peak around the time of the $\mathrm{H}-\mathrm{L}$ transition. For all database entries these peak changes in $\beta_{\mathrm{p}}$ and $f_{\mathrm{GW}}$, normalized by $\tau_{\mathrm{E}}$, are shown in figures $9(a)$ and (b). The values are lower for those cases that gradually rampdown the input power (i.e. the power at the HL transition is smaller with respect to the power at the start of the termination) or those that have a shorter $\mathrm{H}$-mode phase with respect to the current ramp-down. As discussed above, longer H-mode phases, usually lead to high values of $\beta_{\mathrm{p}}$ and $f_{\mathrm{GW}}$, just prior to the $\mathrm{H}-\mathrm{L}$ transition. Typical values are $0.05<\tau_{\mathrm{E}} \times \mathrm{ld} \beta_{\mathrm{p}} /$ $\mathrm{d} t \mid<0.85$ and $0.06<\tau_{\mathrm{E}} \times\left|\mathrm{d} f_{\mathrm{GW}} / \mathrm{d} t\right|<0.60$, which can be compared to those assumed in the modelled ITER cases (see figure 9). The assumed ITER values for the HL transition lie on the upper end of the experimental cloud which is reasonable but could be relaxed in future modelling. The normalization by $\tau_{\mathrm{E}}$, being the characteristic time of the $\mathrm{H}-\mathrm{L}$ transition process, allows a comparison between the various devices. But the figure does not provide information of the actual limit for ITER. $\tau_{\mathrm{E}}$ is not the characteristic time that sets the allowed change in $\beta_{\mathrm{p}}$ with respect to the radial position control. Here 

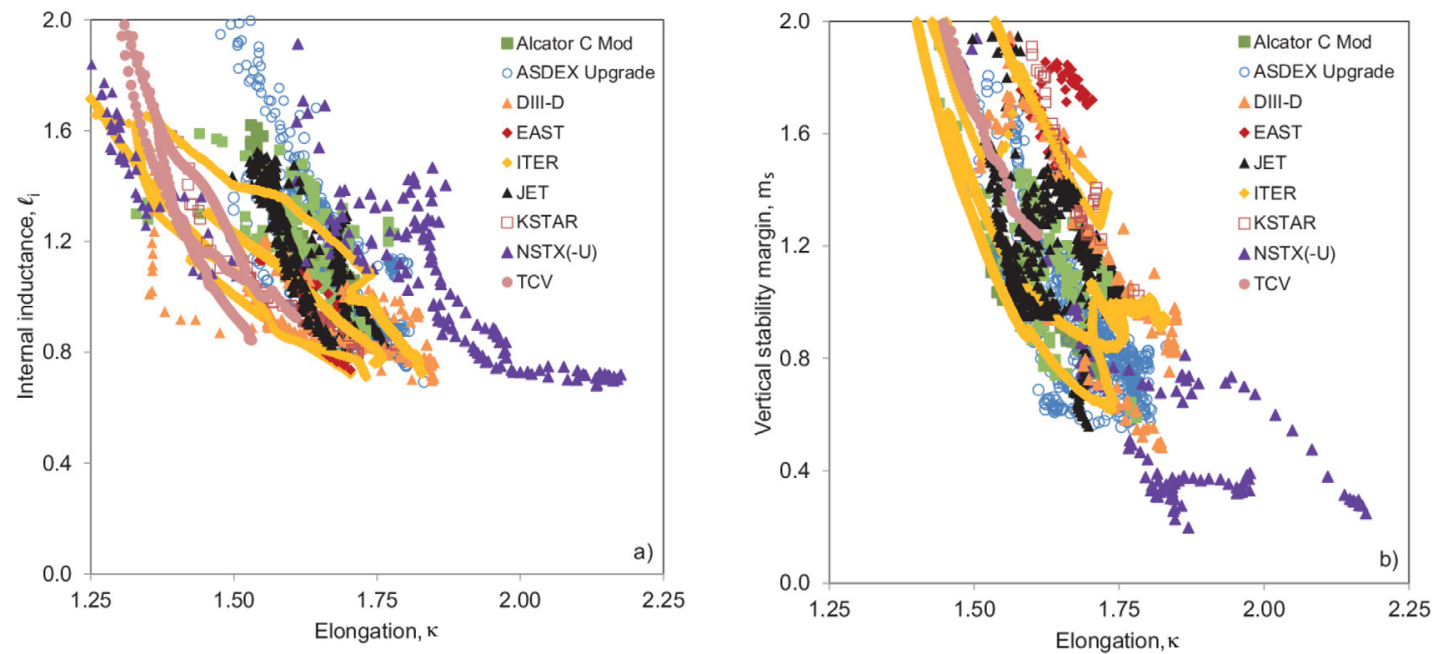

Figure 4. (a) The traces in the $\kappa-\ell_{\mathrm{i}}$ operational space. (b) The marginal stability parameter, $m_{s}$, during the termination of all database entries.

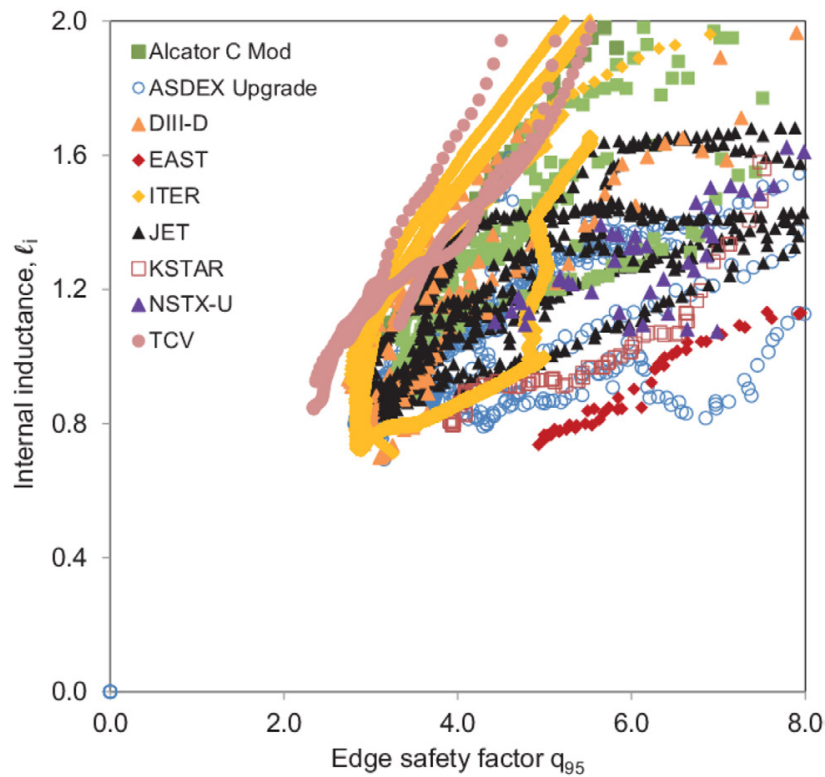

Figure 5. The ramp-down trajectories of each entry in $\ell_{\mathrm{i}}-q$ space. Note that only the NSTX-U entry is visible on this graph.

$\tau_{\mathrm{pc}} \times\left|\mathrm{d} \beta_{\mathrm{p}} / \mathrm{d} t\right|$ should remain below a maximum, where $\tau_{\mathrm{pc}}$ is the characteristic response time of the radial position control, determined by the typical poloidal field coil response and the penetration of the field provided by these coils through the vessel. At ITER, $\tau_{\mathrm{pc}}$ is of the order of several seconds, dominated by the response of the poloidal field coil system, whilst in most present day devices it may be determined by the vessel penetration time. Of course the maximum will depend on details, such as the plasma proximity to the inner wall, at the time of the $\mathrm{H}-\mathrm{L}$ transition, and larger values are allowed for smaller plasmas. Radial and VS control was assessed and maintained in all 4 modelled ITER terminations.

The H-L transition duration was determined by calculating the FWHM (i.e. full width at half maximum) of the time derivative of $\beta_{\mathrm{p}}$, over the transition. Figure $9(c)$ shows that for all devices the duration lasted between $1.5-3 \times \tau_{\mathrm{E}}$. The shortest transitions were found for those entries that had the transition

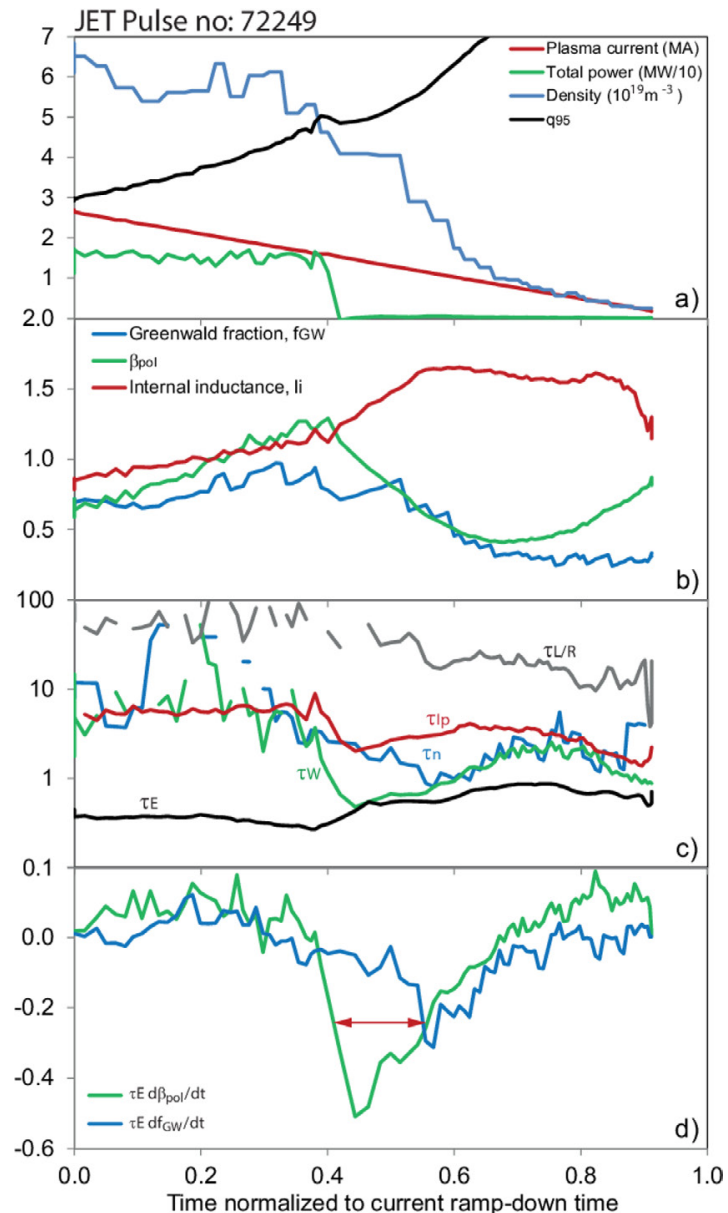

Figure 6. An example of a JET termination (Pulse number 72249), that applies auxiliary power, and keeps $\mathrm{H}$-mode confinement, up to 0.4 of the current ramp-down. (a) The plasma current, $I_{\mathrm{p}}$, the total input power, $P_{\text {tot }}$, the volume averaged electron density and $q_{95}$. (b) The Greenwald fraction, $f_{\mathrm{GW}}$, the poloidal $\beta$, and the internal inductance, $\ell_{\mathrm{i}}$. (c) The decay times, for the plasma current $\tau_{\mathrm{Ip}}$, density, $\tau_{n}$ and energy, $\tau_{W}$, compared to the energy confinement time, $\tau_{\mathrm{E}}$, and the $L / R$ time, $\tau_{L / R}$, all in units of seconds. (d) The time derivative of $\beta_{\mathrm{p}}$ and $f_{\mathrm{GW}}$, normalized to the averaged energy confinement time. 

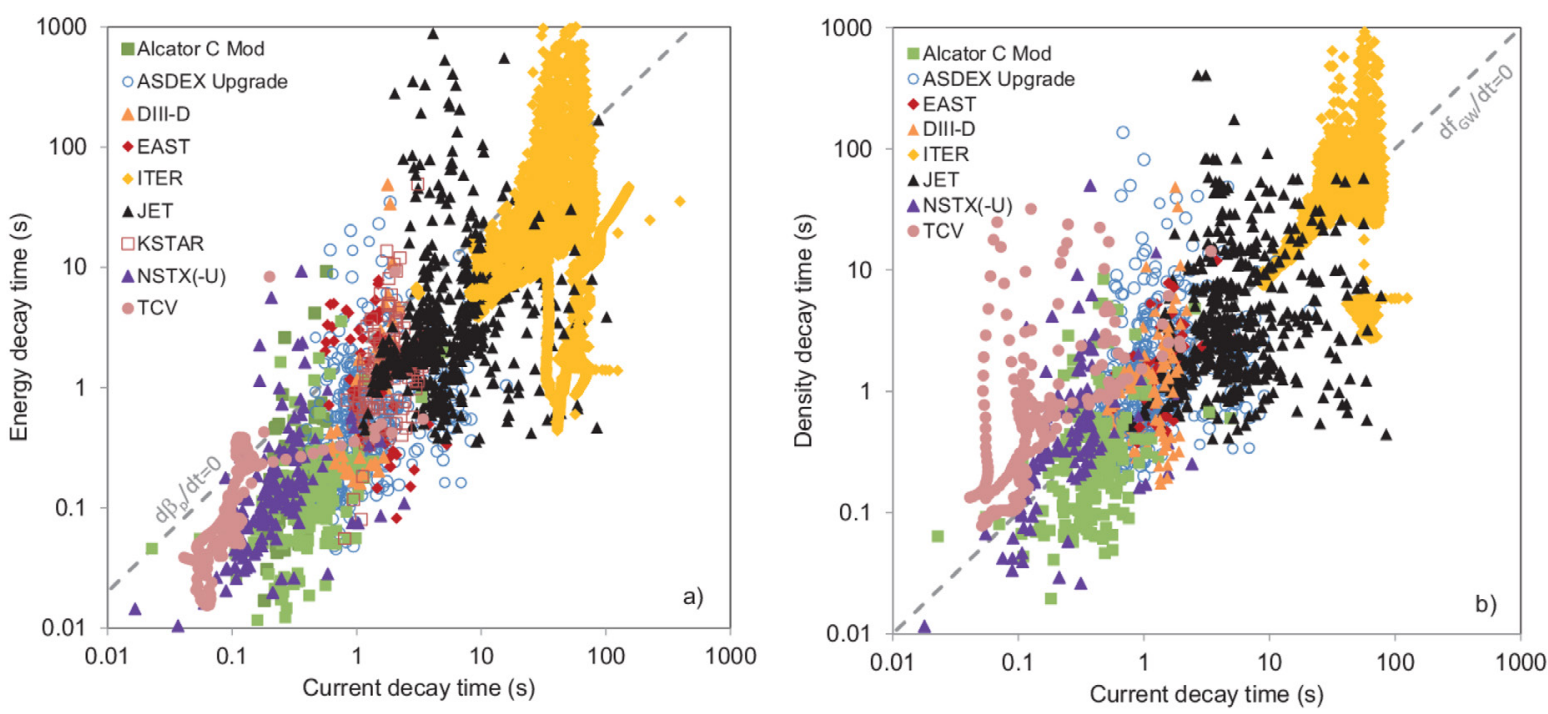

Figure 7. (a) The energy decay time compared to the current decay time for each time step during the termination. Below the dashed line $\beta_{\mathrm{p}}$ decreases. (b) The density decay time compared to the current decay time for each time step during the termination. Below the dashed line, $f_{\mathrm{GW}}$ decreases.
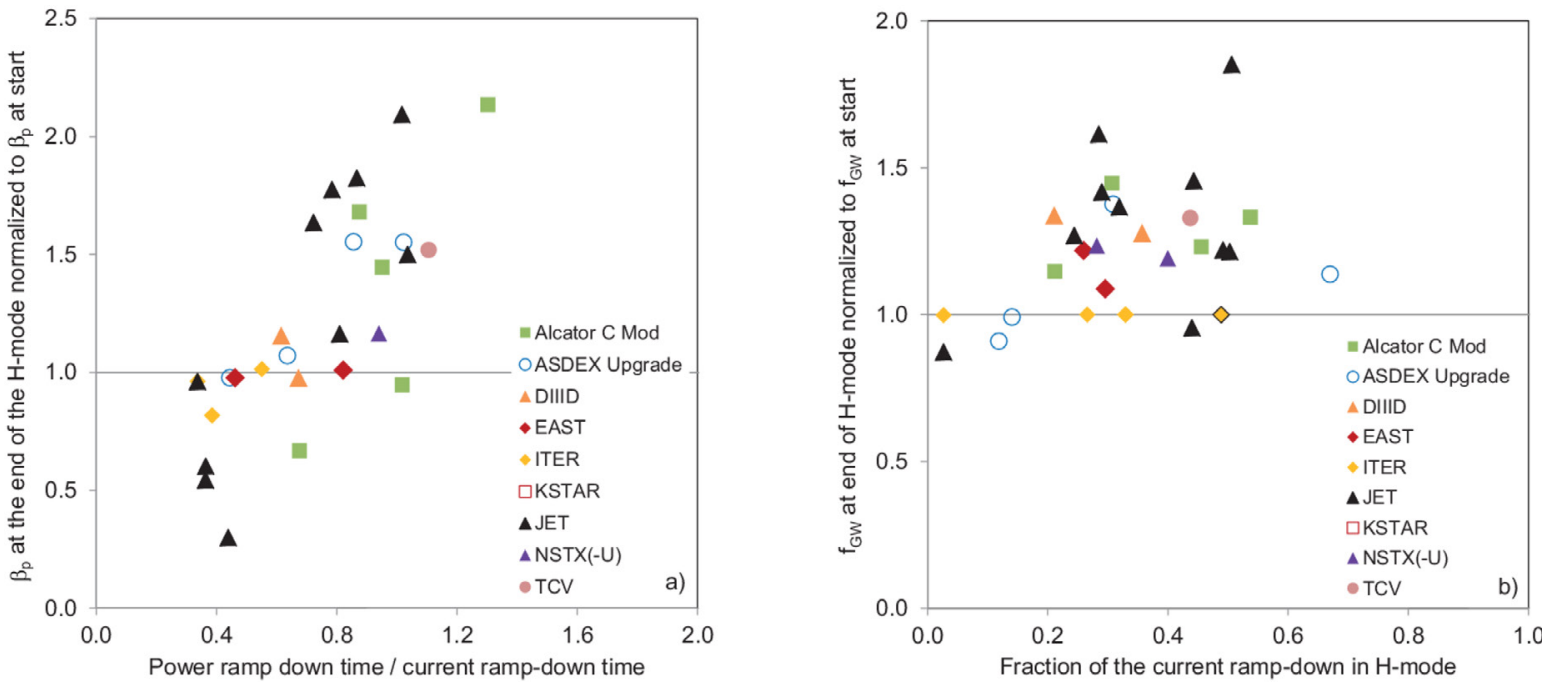

Figure 8. (a) The change in $\beta_{\mathrm{p}}$ over the first part of the termination phase, up to the $\mathrm{H}-\mathrm{L}$ back transition versus the ratio of power rampdown time to current ramp-down time. (b) The change in $f_{\mathrm{GW}}$ up $\mathrm{H}-\mathrm{L}$ back transition, as a fraction of the $f_{\mathrm{GW}}$ at the start of the current ramp-down, versus of the fraction of the current ramp-down kept in H-mode. Note that the modelled ITER cases simply assume that $\mathrm{f}_{\mathrm{GW}}$ remains constant.

later in the termination, at a higher value $\beta_{\mathrm{p}}$ prior to the transition. Slower, and thus softer, transitions are found for those cases that ramped down the input power rather than to step it down. This suggests the need for development of a control scheme to handle the $\mathrm{H}-\mathrm{L}$ transition by preparing the plasma beforehand.

\subsection{The internal inductance dynamics}

The development of $\ell_{\mathrm{i}}$, relevant to VS, is related to both the current ramp-down rate and the $\tau_{L / R}$. Figure 3 has been shown that the latter can vary significantly over the duration of the termination. The current decay time can be compared with the $\tau_{L / R}$ (here averaged over the first half of the current ramp-down), as shown in figure $2(b)$. For the case of a natural current decay in ITER, this ratio is, as expected, near unity. Also a few of the Alcator C-Mod terminations have a current decay time of the same order as the $L / R$ time. This means that for these cases, the current ramp-down is slower than the resistive time, $\tau_{R}$, which is a fraction $(\sim 0.3-0.5)$ of $\tau_{L / R}$ $[10,11]$. Thus for these cases, the internal current density distribution is in equilibrium and follows the redistribution of the resistivity during the ramp-down (i.e. temperature profile changes). On the other hand, for other entries, the current ramp-down is faster than $\tau_{R}$, and one expects a non-equilibrium situation. Changes in $\ell_{\mathrm{i}}$ are driven by the current rampdown itself. The faster, more typical, ITER terminations fall in the last category. Figure 10 shows the absolute change in $\ell_{\mathrm{i}}$, over the first half of the current ramp-down, as a function of the current ramp-down time normalized to $\tau_{L / R}$. This implies 

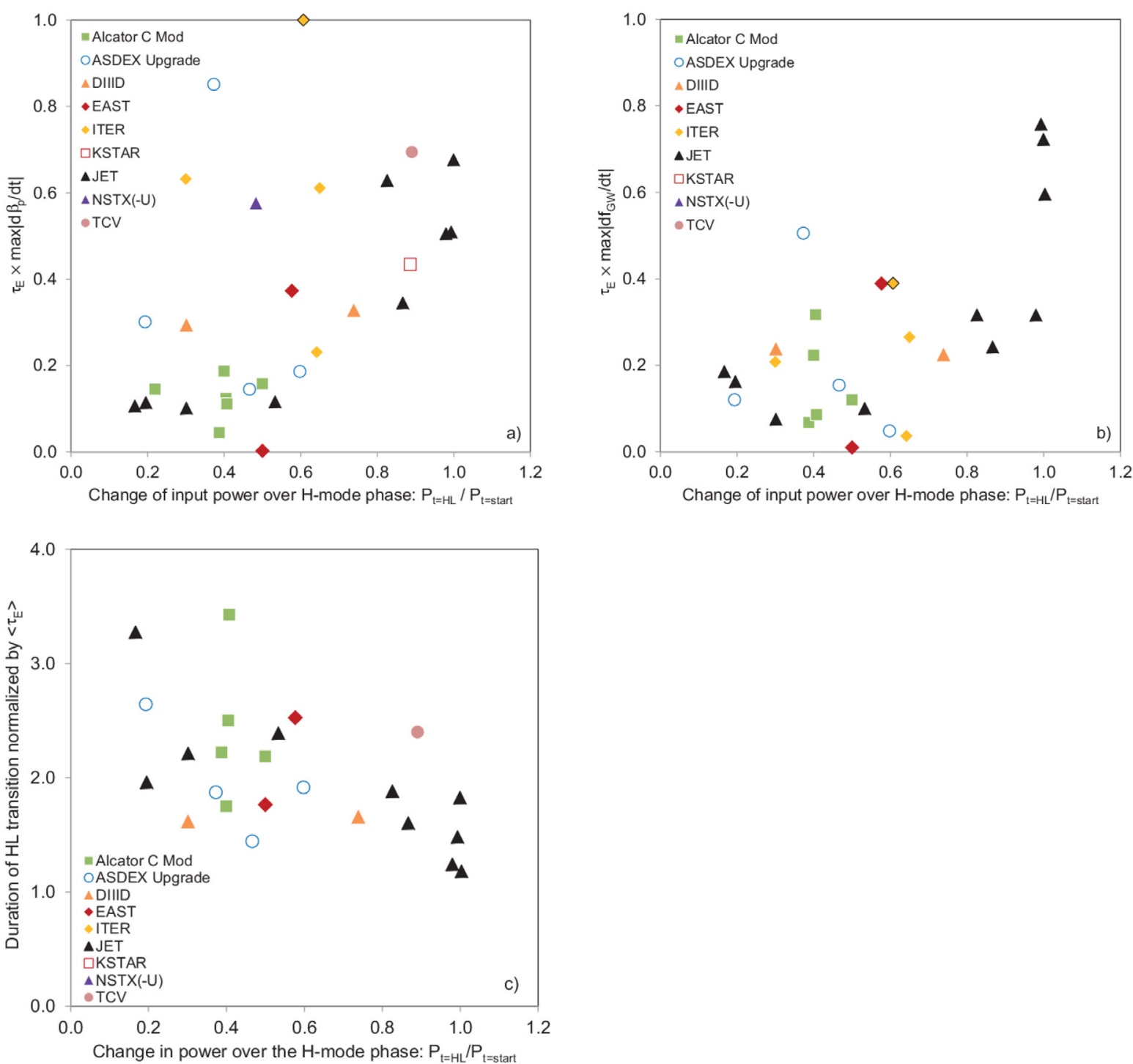

Figure 9. (a) The peak change (i.e. decrease) in $\beta_{\mathrm{p}}$, at the time of the $\mathrm{H}-\mathrm{L}$ transition, normalized to $\left\langle\tau_{\mathrm{E}}\right\rangle$ (averaged over the duration of the $\mathrm{H}-\mathrm{L}$ transition). (b) The peak change in $f_{\mathrm{GW}}$, at the time of the $\mathrm{H}-\mathrm{L}$ transition, again normalized to the same $\left\langle\tau_{\mathrm{E}}\right\rangle$. Note that in both graphs the ITER points represent assumed changes. (c) The duration of the $\mathrm{H}$ to $\mathrm{L}$ transition, defined as the FWHM of $\mathrm{d} \beta_{\mathrm{p}} / \mathrm{d} t$, normalized to $\left\langle\tau_{\mathrm{E}}\right\rangle$.

the current ramp-down rate should be an effective parameter to control the VS in ITER.

\section{Power balance and radiation during the termination}

The fastest changes in plasma energy shown in figure 7, those at the time of the $\mathrm{H}-\mathrm{L}$ transition, are of the order of the energy confinement time, $\tau_{\mathrm{E}}$. In the example shown in figure 6 , the energy decay time, $\tau_{W}$, touches the value of $\tau_{\mathrm{E}}$, during the transition. At this time the loss power is dominated by the $\mathrm{d} W /$ $\mathrm{d} t$ component. Here, the loss power is the power conducted through the plasma separatrix, being the total absorbed auxiliary heating plus $\alpha$-power and, especially for terminations, the energy decay power, $\mathrm{d} W / \mathrm{d} t$, with the bulk radiative power subtracted. Prior and after the $\mathrm{H}-\mathrm{L}$ transition, the fraction of the $\mathrm{d} W / \mathrm{d} t$ contribution to the loss power is much smaller. The typical energy decay time, $\tau_{W}$, during these phases is larger than the energy confinement time. High levels of radiation can affect the stability of the termination and result in disruptions [15]. As it will decrease the loss power, it is likely the radiative power is also able to affect the $\mathrm{H}-\mathrm{L}$ back transition.

\subsection{The $L H$ threshold and ELMs}

The database entries have a varied level of input power with respect of the $\mathrm{H}$-mode threshold, $P_{\mathrm{IN}} / P_{\mathrm{LH}}$ at the start of the termination. Assuming the standard $\mathrm{H}$-mode power threshold [16], one find that most cases in the database, $P_{\mathrm{IN}} / P_{\mathrm{LH}}$ falls between 1 and 2, as shown in figure 11. Some operate at higher ratios above 2 . The latter is often motivated by operation at a higher Type I edge localized mode (ELM) frequency to control the impurity content in devices with high $Z$ metal PFC [17].

The database was not suited to show influence of radiation on the H-mode and ELM dynamics. A better picture is presented using a series of 121 near identical discharges that were carried out at JET (JET Pulse numbers: 83623-83777). 


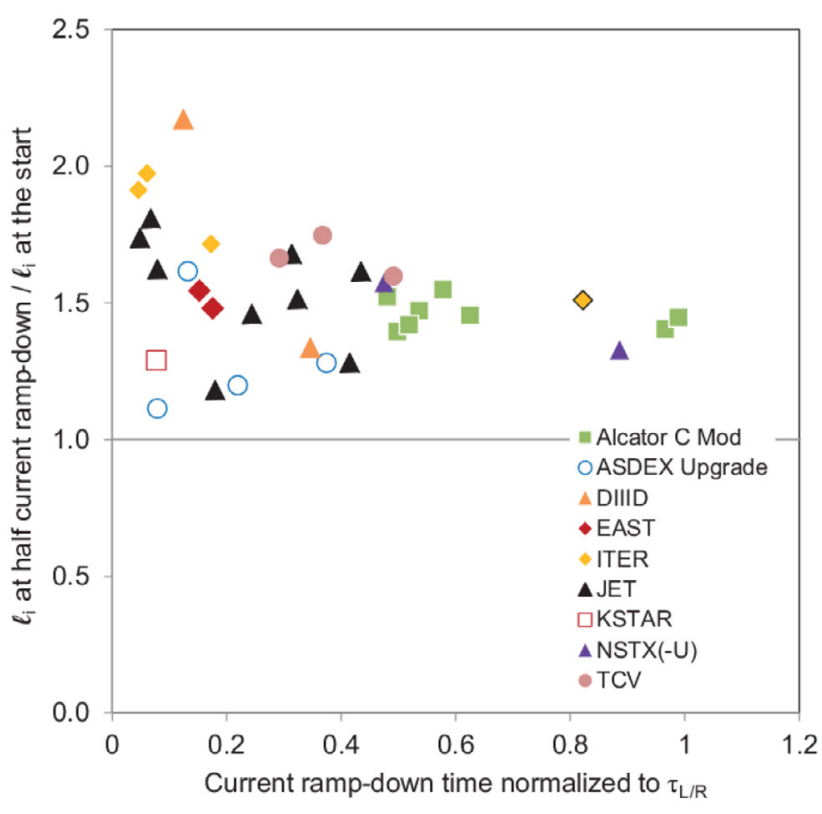

Figure 10. Existence diagram of the relative change in $\ell_{\mathrm{i}}$ over half the current ramp-down versus the ratio of the averaged current decay time and $\tau_{L / R}$.

All operated in H-mode at $I_{\mathrm{p}}=2.0 \mathrm{MW}, q_{95}=3.4$ with an auxiliary neutral beam (NB) heating of $P_{\mathrm{NB}} \sim 11 \mathrm{MW}$. At the start of the current ramp-down, the auxiliary heating power was stepped down to zero. This scenario causes a much more rapid and uncontrolled $\mathrm{H}-\mathrm{L}$ back transition than those proposed for ITER. Such rapid termination of auxiliary heating has been studied for ITER scenarios [6], although those considered in the database have a smoother ramp-down.

The plasma remained in Type I ELMy H-mode, for as long as the loss power, through the separatrix, remains above the $\mathrm{H}$-mode threshold, but eventually an $\mathrm{H}-\mathrm{L}$ back transition takes place. Although the terminations are programmed identically, the H-mode dynamics varied considerably due to small variations in the impurity levels and radiative power. After the rapid power ramp-down, the plasma remains typically 1 to 2 energy confinement times in H-mode. Figure 12(a) shows that the duration that the plasma stays in $\mathrm{H}$-mode, $\Delta t_{\mathrm{H}}$, is reduced for higher radiative power fractions, $f_{\mathrm{RAD}}$. For this scenario, the analysis breaks down for radiative power fractions of the order of $f_{\mathrm{RAD}} \sim 0.35$, as the $\mathrm{H}-\mathrm{L}$ transition becomes too ambiguous and $\Delta t_{\mathrm{H}}$, too short accurately determine. Note that radiation has not been included in the H-mode power threshold scaling [16]. In these specific cases, there is a significant contribution of the radiative power to the energy decay, thus affecting $\tau_{W}$, as shown in figure 12(b). Radiative energy losses obviously do not contribute to the separatrix loss power. Only the fraction of the $\mathrm{d} W / \mathrm{d} t$ component that is convected through the separatrix contributes to the loss power that could keep the plasma in H-mode. Similar considerations also affect the behavior of the ELMs, with lower ELM frequencies and ELM free phases, towards the end of the H-mode phase, becoming more likely at higher radiative fractions. Moreover, the plasma current ramp-down itself may also affect ELM stability. A slower power ramp-down will provide more control over the $\mathrm{H}-\mathrm{L}$ back transition. And, the exact trends shown here depend on

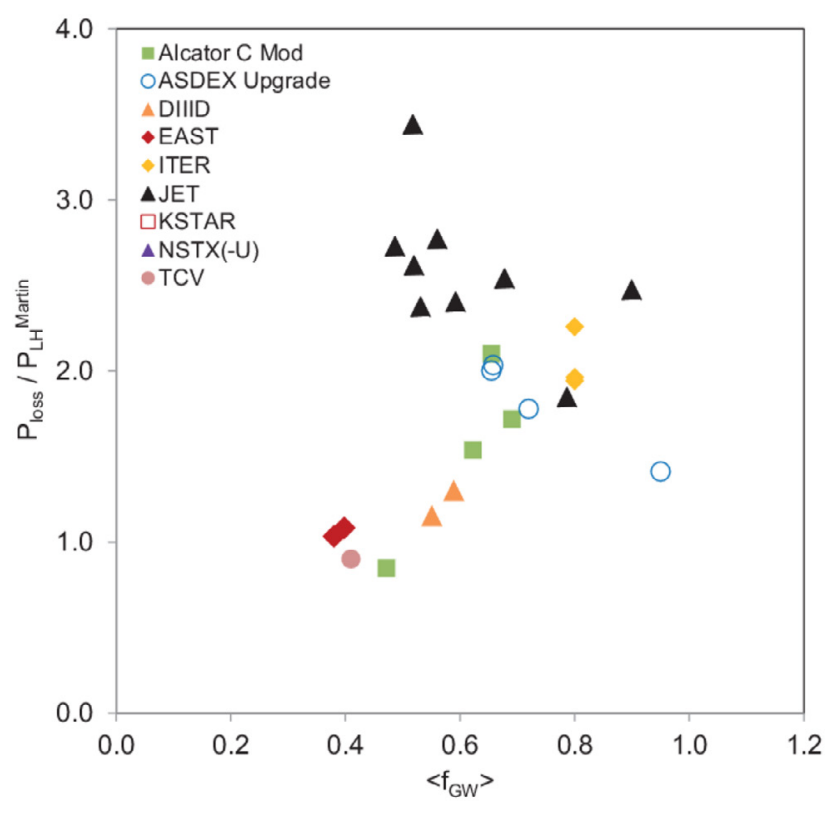

Figure 11. The ratio of the input power to the H-mode threshold power, as defined by [16], at the start of the current ramp-down. Only terminations that are in $\mathrm{H}$-mode at the start of the termination are included.

various other plasma parameters. But the results show that radiation will affect this transition, and can result, at high radiation levels, in low frequency ELMs, that destabilize the termination and influence the impurity control. ITER may have to adjust its termination scenario, by means of tuning the auxiliary heating power, depending on the radiation levels to optimally control the $\mathrm{H}-\mathrm{L}$ transition.

\subsection{Radiative power and impurities}

From the discussion above, a self-consistent assumption for the impurity and radiation powers is important when modelling ITER termination scenarios. Typical radiation levels found during the terminations in the experimental database can be compared with those assumed for the modelled ITER terminations. One way to compare the amount of radiation during the termination is by normalizing it to the total input power. Figure 13, shows the radiative power fractions at the start of the termination, with a wide variation between 0.4 and 0.8. Later during the current ramp-down, and especially after the auxiliary power ramp-down, the values may vary significantly. The radiative power fraction, $f_{\mathrm{RAD}}$, is relevant for the stability of the termination. Radiation too high compared to the heating power, can lead to disruptions. However, the radiative fraction may vary significantly within a single device, from discharge to discharge, and it may not be practical to use it for comparison. Another way is to compare radiation values is by normalizing it to the particle density, as the radiation is thought to scale with the particle density times the impurity density. The latter is often converted into a scaling with the square of the density, assuming a constant impurity content [18]. In figure 14, the radiation power, per plasma volume, is plotted versus the electron density, for a number of terminations in the database. It shows that the experimental data scales 

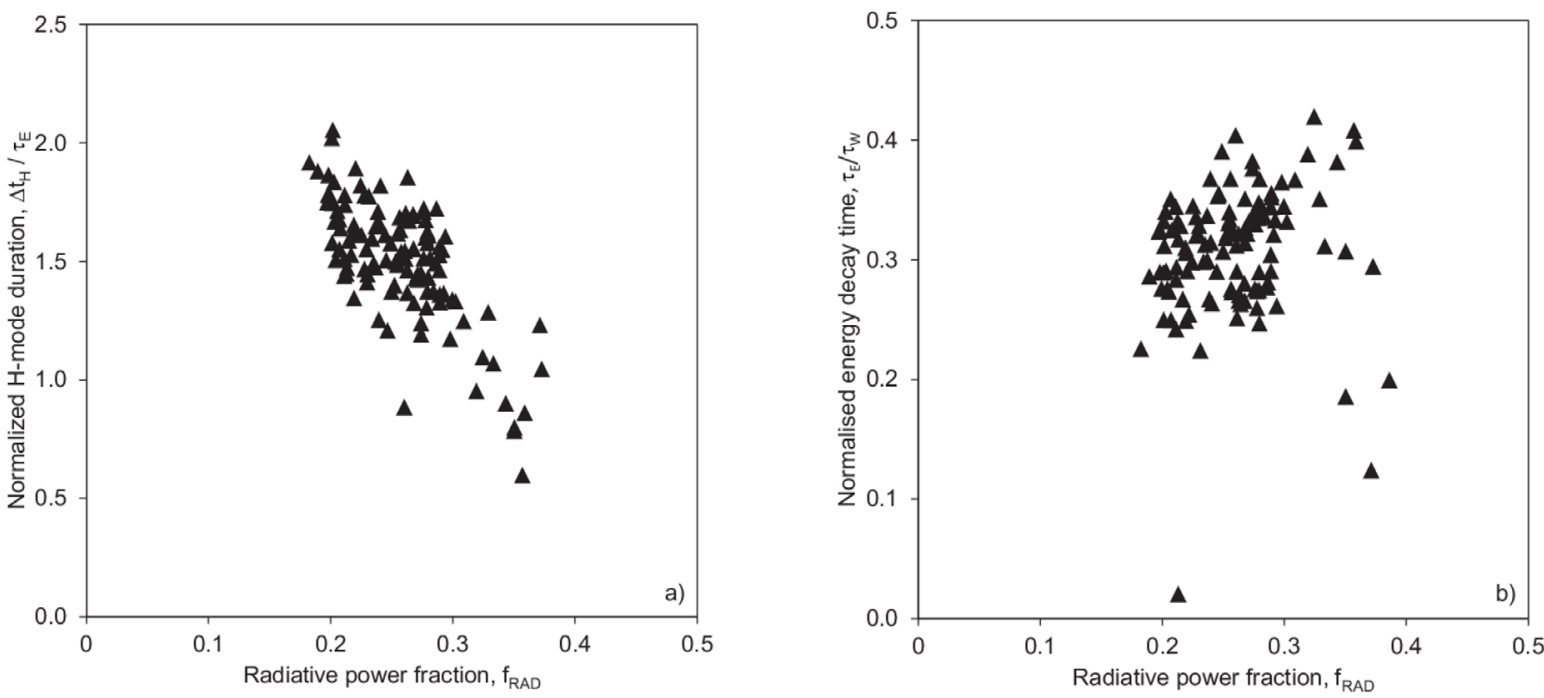

Figure 12. A series of 121 near identical discharges that were carried out at JET (JET Pulse numbers from 83623 to 83777 ). All operated in H-mode at $I_{\mathrm{p}}=2.0 \mathrm{MW}, q_{95}=3.4$ with an auxiliary neutral beam (NB) heating of $P_{\mathrm{NB}} \sim 11 \mathrm{MW}$. At the start of the current ramp-down, the auxiliary heating power was stepped down to zero, after which the plasma remains in $\mathrm{H}$-mode for a time $\Delta t_{\mathrm{H}}$. $(a) \Delta t_{\mathrm{H}}$, normalized to the energy confinement time, $\tau_{\mathrm{E}}$, as a function of the radiative power. (b) The inverse ratio of the average energy decay time and the steadystate energy confinement time at the start of the termination, $\tau_{\mathrm{E}} / \tau_{W}$, as a function of the radiative power fraction.

to leading order with the square of the density. Note that the scaling extends over large density range over three decades. Some individual entries follow scaling (with the square of the density) for the entire termination from high densities at the start of the current ramp-down until the end at densities 2 orders or magnitude lower. Deviations are predominantly due to differences in impurity content. Some ASDEX-Upgrade, JET and Alcator C-MOD discharges show strong outliers that can be attributed to the presence of higher levels of high- $Z$ impurities or the development of radiation instabilities, such as MARFEs, during these terminations.

A previous scaling for radiative power, predominantly from low- $Z$ impurity line-emission, scaled with the plasma outer area [18]. Here a satisfactory scaling is found when the radiation is normalized with the entire volume. This is understandable because the examples in the database are not necessarily dominated by edge line-emission. The database provides examples of devices with both carbon and metal walls but does not contain examples that utilize low- $Z$ impurity seeding. The modelled ITER cases usually start the termination with a dominant contribution of bremsstrahlung and a lower level of low- $Z$ line radiation. Moreover, the high- $Z$ line-emission is usually emitted from the hotter core of the plasma. A radiation quality or radiation cooling quality [19] can be defined as the radiative power (MW) normalized to the density squared per unit volume. The experimental values in figure 14 correspond to radiation cooling qualities within the range of 0.1 and $1 \times 10^{-40}\left(\mathrm{MW} \mathrm{m}^{-3}\right)$, comparable to what is usually found in tokamaks $[19,20]$.

Notably, the modelled ITER cases, lie well below the experimental values, suggesting a possible underestimation of the radiative power. The radiation from ITER plasmas during the high temperature burn phase will be dominated by Bremsstrahlung. Higher temperatures in ITER plasmas

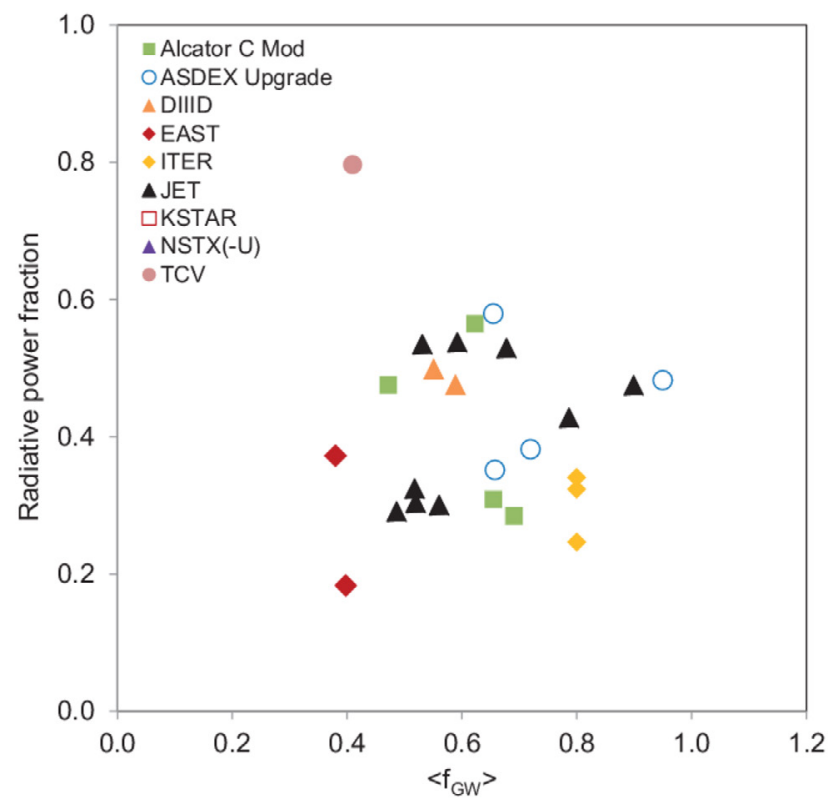

Figure 13. Radiative power fraction at the start of each termination in the database versus the Greenwald fraction, $f_{\mathrm{GW}}$ at the same time. Radiation data were not available for all entries.

yield lower levels of line-emission, for similar levels of impurities. Hence, at the start of the termination from burn, the modelled cases are not expected to match the experiment basis. However, it is not evident if later in the current rampdown, the assumed radiation in the modelled ITER terminations is self-consistent. Assuming higher levels of impurities and radiation may however, increase the radiative power fraction and yield unstable terminations. Self-consistent calculations of the impurity content and resulting radiation should be taken into account when modelling ITER termination scenarios $[21,22]$. 


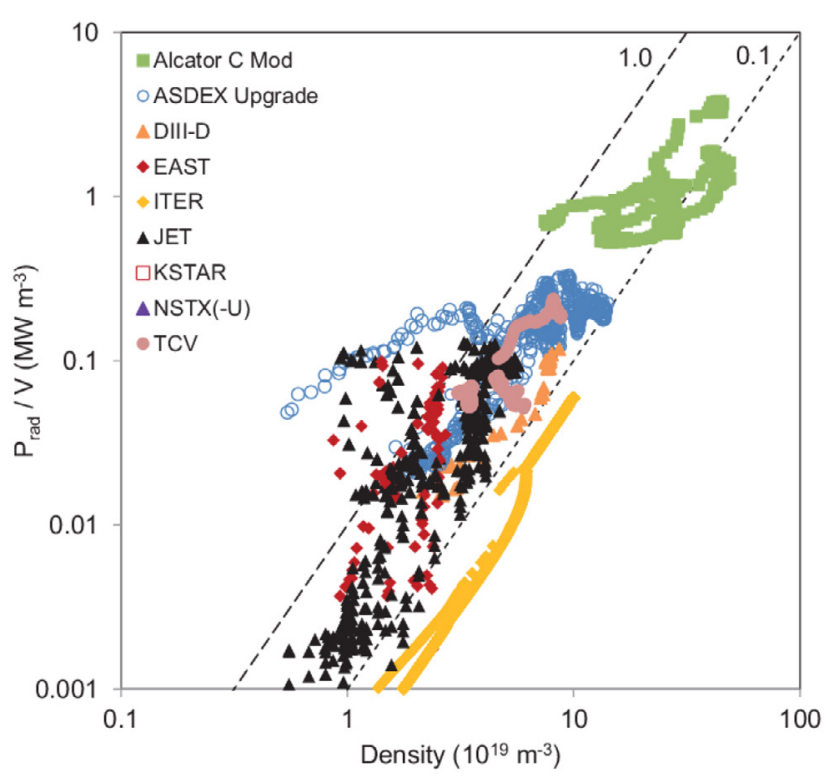

Figure 14. Radiative power over the entire termination, normalized to the plasma volume, versus the electron density. Not for all entries radiation data were available. The long and short dashed lines indicate the radiation quality values of 0.1 and $1.0 \times 10^{-40}$ $\left(\mathrm{MW} \mathrm{m}^{3}\right)$, respectively.

\section{Recommendations}

The database, built using a selected set of experimental termination cases, showed many similarities in the particle dynamics and current density behavior. Differences are found in relation to the specific control and heating capabilities of each device. Relevant for ITER is the capacity to maintain vertical, radial position, and shape control during the termination, especially at the time of the relatively fast $\mathrm{H}-\mathrm{L}$ transition. The task is to show whether the specific ITER design features allow a stable well-controlled termination. This is a joint effort in control, exception handling development and physics modelling [13, $23,24]$. The results from this analysis can be used to better prescribe the inputs for the detailed modelling and preparation of ITER termination scenarios. Models capable of real-time simulation are being developed and should further help in obtaining robust termination phases in ITER [25].

Present day devices can provide significant input power during a large fraction of the current ramp-down, keeping the plasma in $\mathrm{H}$-mode and slowing down the increase in $\ell_{\mathrm{i}}$. The auxiliary power available at ITER limits this capability. Especially for a termination with a significant fraction of $\alpha$-power, the ramp-down of the input power compared to the current is relatively fast, hence it is difficult to maintain H-mode and control the $\mathrm{H}-\mathrm{L}$ transition. To maintain VS, a strong reduction in elongation during the ITER current ramp-down is essential. As a consequence, ITER terminations remain longer than present day devices at lower $\mathrm{q}_{95}$ and trace the upper boundary of the $\ell_{\mathrm{i}}-q$ stability diagram. The impact on the MHD stability for such terminations needs to be assessed. This situation may be different when terminating an ITER H-mode without a larger fraction of $\alpha$-power, for example at $I_{\mathrm{p}}=7.5 \mathrm{MA}$ and $B=2.65 \mathrm{~T}$.

Particle exhaust and control of the density is an important aspect of a termination. The decay of the H-mode density can be seen as a change in the overall particle confinement or one could view it as a decay of specifically the pedestal density, when the current is ramped down. From the $\mathrm{H}_{98 \mathrm{y} 2}$ confinement scaling one would expect the energy confinement to decay proportional to $I_{\mathrm{p}}$ [26]. The pedestal pressure is expected to decay by $\sim I_{\mathrm{p}}^{2}$, hence the density and temperature can be assumed individually to scale approximately linearly with $I_{\mathrm{p}}$ [27]. Scaling of parameters can be used to determine their relative changes, such as the behavior of the density or pedestal pressure with the plasma current. But these scalings developed for stationary plasmas should be valid for the non-steady state current ramp-down phase. For the pedestal specifically, ramping down the plasma current could affect its stability and hence its behavior. The ramp-down of the heating power during the final part of the $\mathrm{H}$-mode phase, will reduce the type I ELM frequency, affecting particle exhaust and hence could explain the density behavior. The density, during the H-mode phase, was often found to decay more slowly than the plasma current. The consequential rise in $\mathrm{f}_{\mathrm{GW}}$ during the current ramp-down will limit the duration of the H-mode phase, especially when terminating from a high $f_{\mathrm{GW}}$ scenarios. ITER has to put a stronger emphasis on the reduction in elongation and controlling the current ramp-down rate to provide VS. A better understanding of the behavior of the H-mode pedestal in dynamic situations, like during a ramp-down of the power, plasma $\beta$ and plasma current, should be developed to prepare for more robust ITER terminations.

ITER terminations will benefit from controlled H-L transitions and this phase should be studied experimentally in more detail and properly modelled. The database cases all showed an H-L transition duration of the order of a several times $\tau_{\mathrm{E}}$. Radiative power should be accounted for in the power balance. The timing $\mathrm{H}-\mathrm{L}$ transition depends both on the core radiation and value of $\mathrm{dW} / \mathrm{dt}$ convected through the separatrix. The radiation levels relate to the methods of density and impurity control. Self-consistent calculations of the impurity content and resulting radiation should be taken into account when modelling ITER termination scenarios. The exit from H-mode, with a possible phase of low frequency type I ELMs or a longer final ELM free phase, needs to be carefully designed and modelled, not only with respect to the radial and VS control, but also with respect to density, impurity and the ELM and control [21].

Importantly, the dynamics of the changes (ramp-down) of magnetic and thermal energy, are coupled, though characteristic time scales, such as energy confinement time, resistive time, density decay, which do not scale the same from device to device. Thus a full integrated assessment of the robustness of proposed ITER terminations can only be performed by detailed modelling of the plasma dynamics and control.

\section{Acknowledgments}

ITER is a Nuclear Facility INB-174. This material is based in work using; the National Magnetic Confinement Fusion Program of China (No. 2015GB102000), DoE Office of Science user Facilities Alcator C-Mod, NSTX, and DIII-D, 
supported by DoE Awards DE-FC02-99ER54512, DE-AC0276CH03073, DE-FC02-04ER54698, respectively, the MSIP Fusion Program KSTAR, the framework of the EUROfusion Consortium and received funding from the Euratom research and training program 2014-2018 under grant agreement No 633053. The views and opinions expressed herein do not necessarily reflect those of the ITER Organization or the European Commission.

\section{ORCID iDs}

C. Kessel (1) https://orcid.org/0000-0002-2072-1134

F. Poli (i) https://orcid.org/0000-0003-3959-4371

\section{References}

[1] Sips A.C.C. et al 2009 Nucl. Fusion 49085015

[2] Politzer P.A. et al 2010 Nucl. Fusion 50135011

[3] Kessel C.E. et al 2013 Nucl. Fusion 53093021

[4] Lehnen M. et al 2015 J. Nucl. Mater. 463748

[5] Gribov Y. et al 2016 Controlled emergency plasma termination in ITER Proc. 43rd EPS Conf. on Plasma Physics (Leuven, Belgium, 4-8 July 2016) (http://ocs.ciemat.es/EPS2016PAP/ pdf/P4.066.pdf)

[6] Loarte A. et al 2014 Nucl. Fusion 54123014

[7] Casper T.A. et al 2014 Nucl. Fusion 54013005
[8] Kim S.H. et al 2016 Nucl. Fusion 56126002

[9] Khayrutdinov R.R. and Lukash V.E. 1993 J. Comput. Phys. 109193

[10] Mikkelsen D.R. 1989 Phys. Fluids B 1333

[11] Luce T.C. et al 2005 Nucl. Fusion 45 S86

[12] Humphreys D. et al 2009 Nucl. Fusion 49115003

[13] Humphreys D. et al 2016 Plasma Control Studies Using DIII-D Design Tools in Support of ITER Preprint: 2016 IAEA Fusion Energy Conf. (Kyoto, Japan) [EX/P6-37]

[14] Greenwald M. 2002 Plasma Phys. Control. Fusion 44 R27

[15] de Vries P.C. et al 2014 Phys. Plasmas 21056101

[16] Martin Y.R. et al 2008 J. Phys. Conf. Ser. 123012033

[17] Loarte A. et al 2014 Nucl. Fusion 54033007

[18] Matthews G.F. et al 1997 J. Nucl. Mater. 241-3 450

[19] Telesca G. et al 2000 Nucl. Fusion 401845

[20] Devynck P. et al 2014 Plasma Phys. Control. Fusion 56075026

[21] Loarte A. et al 2016 Evaluation of tungsten transport and concentration control in ITER scenarios Preprint: 2016 IAEA Fusion Energy Conf. (Kyoto, Japan) [PPC/2-1]

[22] Koechl F. et al 2016 Evolution and control of tungsten transport in the termination Phase of JET H-mode discharges and implications for ITER Preprint: 2016 IAEA Fusion Energy Conf. (Kyoto, Japan) [EX/P6-15]

[23] Humphreys D. 2015 et al Phys. Plasmas 22021806

[24] Snipes J.A. et al 2017 Nucl. Fusion 57125001

[25] Teplukhina A. et al 2017 Plasma Phys. Control. Fusion 59124004

[26] McDonald D.C. et al 2007 Nucl. Fusion 47147

[27] Snyder P.B. et al 2009 Phys. Plasmas 16056118

[28] Litaudon X. et al 2017 Overview of the JET results in support to ITER Nucl. Fusion $\mathbf{5 7} 102001$ 